\title{
Microbial predation accelerates granulation and modulates microbial community composition
}

Siew Herng Chan ${ }^{1,2+}$, Muhammad Hafiz Ismail ${ }^{1,3+}$, Chuan Hao Tan ${ }^{1,4}$, Scott A. Rice, $1,35^{*}$ and Diane McDougald ${ }^{1,5^{*}}$

\begin{abstract}
Background: Bacterial communities are responsible for biological nutrient removal and flocculation in engineered systems such as activated floccular sludge. Predators such as bacteriophage and protozoa exert significant predation pressure and cause bacterial mortality within these communities. However, the roles of bacteriophage and protozoan predation in impacting granulation process remain limited. Recent studies hypothesised that protozoa, particularly sessile ciliates, could have an important role in granulation as these ciliates were often observed in high abundance on surfaces of granules. Bacteriophages were hypothesized to contribute to granular stability through bacteriophage-mediated extracellular DNA release by lysing bacterial cells. This current study investigated the bacteriophage and protozoan communities throughout the granulation process. In addition, the importance of protozoan predation during granulation was also determined through chemical killing of protozoa in the floccular sludge.

Results: Four independent bioreactors seeded with activated floccular sludge were operated for aerobic granulation for 11 weeks. Changes in the phage, protozoa and bacterial communities were characterized throughout the granulation process. The filamentous phage, Inoviridae, increased in abundance at the initiation phase of granulation. However, the abundance shifted towards lytic phages during the maturation phase. In contrast, the abundance and diversity of protozoa decreased initially, possibly due to the reduction in settling time and subsequent washout. Upon the formation of granules, ciliated protozoa from the class Oligohymenophorea were the dominant group of protozoa based on metacommunity analysis. These protozoa had a strong, positivecorrelation with the initial formation of compact aggregates prior to granule development. Furthermore, chemical inhibition of these ciliates in the floccular sludge delayed the initiation of granule formation. Analysis of the bacterial communities in the thiram treated sludge demonstrated that the recovery of 'Candidatus Accumulibacter' was positively correlated with the formation of compact aggregates and granules.

(Continued on next page)
\end{abstract}

\footnotetext{
*Correspondence: rscott@ntu.edu.sg; Diane.McDougald@uts.edu.au

†Siew Herng Chan and Muhammad Hafiz Ismail contributed equally to this work.

${ }^{1}$ Singapore Centre for Environmental Life Sciences Engineering, Nanyang Technological University, 60 Nanyang Drive, Singapore 637551, Singapore Full list of author information is available at the end of the article
} 


\begin{abstract}
(Continued from previous page)
Conclusion: Predation by bacteriophage and protozoa were positively correlated with the formation of aerobic granules. Increases in Inoviridae abundance suggested that filamentous phages may promote the structural formation of granules. Initiation of granules formation was delayed due to an absence of protozoa after chemical treatment. The presence of 'Candidatus Accumulibacter' was necessary for the formation of granules in the absence of protozoa.
\end{abstract}

Keywords: Granulation, Protozoa, Bacteriophage, Metagenomics, Activated sludge, Microbial predation

\section{Background}

Aerobic granular sludge is a complex, human engineered ecosystem consisting of highly diverse and functional microbial communities that are utilized for specific biological functions $[1,2]$. These densely packed biofilm aggregates are typically developed from activated floccular sludge. Using laboratory sequencing batch reactors (SBRs), the formation of aerobic granules from flocs has been improved with the concomitant increased understanding of the effects of operating conditions such as hydrodynamic shear force, settling time, hydraulic retention time and discharging time [3-7].

In contrast to the impact of physical factors, the biological processes that drive granule formation are less well understood. For example, N-acyl-homoserine-lactone (AHL) mediated quorum sensing was found to positively correlate with the formation of granules from floccular sludge [8]. Furthermore, the addition of AHLs to the SBR markedly increased the production of EPS, which mediates contact between bacterial cells $[8,9]$. Other biological factors such as predation have been demonstrated to enhance biofilm formation for several bacterial species [10-12]. Predation on those free-living bacteria may therefore represent a strong pressure selecting for bacteria that are tightly embedded in aggregates of biomass. Bacteriophages are highly abundant in engineered wastewater systems, appear to be active components of activated sludge systems and are able to infect both planktonic and biofilm associated bacterial cells [13-15].

Phage-mediated mortality has the potential to influence the treatment performance of a system through controlling the abundance of key functional groups, leading to their utilisation as a biocontrol strategy to lyse filamentous bacteria that are responsible for bulking in activated sludge $[16,17]$. In contrast, bacteriophage predation in wastewater systems has been demonstrated to cause the collapse of reactors [18] and the failure of bacterial biological processes such as phosphorus removal and nitrification $[18,19]$. Bacteriophage predation has recently been suggested to mediate the release of extracellular DNA via the lysis of bacterial cells, which plays a role in providing structural stability to granules [20].
Protozoa are abundant in activated floccular sludge systems and play an important role in the predation of suspended bacteria, which aids in the clarification of wastewater effluent [21]. In addition, previous studies of aerobic granulation systems demonstrated an abundance of sessile ciliates on the surface of aerobic granules [2124]. Electron microscopy of granular surfaces revealed the attachment of bacteria on the stalks of sessile ciliates [24]. Weber et al. [24] further hypothesised that these sessile ciliates may act as nucleating agents for the attachment of bacteria. Taken together, these studies strongly suggest that protozoan predation may have a role in promoting aerobic granulation. However, the role of protozoa in the formation of aerobic granules remains unclear to date.

Here, bacteriophage, protozoan and bacterial communities were characterized throughout the aerobic granulation process. The dynamics of different bacteriophage families were investigated to elucidate their role in granulation. Additionally, the succession of protozoan communities was tracked during the aerobic granulation process and the inhibition of protozoa was performed to determine the potential role of protozoan predation in driving aerobic granulation. It is hypothesised that protozoan predation can promote the formation of granules through grazing selection pressure and/or via a structural role. Microbial community analysis indicated that there was an increase in the abundance of non-lytic, filamentous Inoviridae bacteriophages during the initiation phase of granulation when compact aggregates were formed. In addition, the abundance and diversity of protozoa decreased significantly during the aerobic granulation process. Our results also demonstrated that the absence of protozoa did not negatively affect the formation of mature granules, although there was a delay in the formation of compact aggregates in the absence of protozoa.

\section{Results}

\section{Development and microscopic observations of aerobic} granular sludge

Activated floccular sludge was used to seed the SBRs, which were operated under conditions optimal for the aerobic granulation process over a period of 11 weeks. The granulation process has five distinct phases: 
floccular, initiation, maturation, maintenance and dispersal [8]. Here, only three phases of floccular, initiation and maturation phases were observed (Fig. 1a).

During Phase I, the floccular biomass had a mean particle size of $51.3 \pm 2.2 \mu \mathrm{m}$ (50th percentile) (Fig. 1b). Aerobic granules are typically defined as dense and compact aggregates characterized by a minimum particle size of $100 \mu \mathrm{m}$ and a $\mathrm{SVI}_{5}$ of $50 \mathrm{~mL} \mathrm{~g}^{-1}$ or less [25]. Initial decreases in settling time from 120 to 56 min resulted in a $10.5 \%$ average loss of biomass (MLSS decreased from $5.0 \pm 0.1$ to $4.1 \pm 0.1 \mathrm{~g} \mathrm{~L}^{-1}$ ) by the end of week 1 (Fig. 1c). The $\mathrm{SVI}_{5}$ of the floccular sludge increased from $190.8 \pm 2.0$ to $221.8 \pm 5.4 \mathrm{~mL} \mathrm{~g}^{-1}$, which indicated poor settling of the floccular sludge (Fig. 1b).

By Phase II, compact aggregates were observed in the floccular sludge at week 4 and the mean particle size was $96.2 \mu \mathrm{m}$ (50th percentile) (Fig. 1b). Subsequent decreases in settling time from 56 to $24 \mathrm{~min}$ did not result in a decrease in overall biomass until week 4 (MLSS increased from $4.9 \pm 0.4$ to $5.1 \pm 0.4 \mathrm{~g} \mathrm{~L}^{-1}$ ) when the sludge biomass entered the Phase II. During weeks 4 to 6 of Phase II, the settling time was reduced from 24 to $5 \mathrm{~min}$, which resulted in an average of $23.7 \%$ loss of biomass (MLSS decreased from $5.1 \pm 0.4$ to $3.9 \pm 0.5 \mathrm{~g} \mathrm{~L}^{-1}$ ) (Fig. 1b). This reduction in settling time also coincided with an increase in mean particle size from $108.5 \pm 6.9$ to $193.0 \pm 16.7 \mu \mathrm{m}$ (50th percentile) (Fig. 1b). In addition, the $\mathrm{SVI}_{5}$ also decreased $44 \%$ from $112.5 \pm 13.2$ to $63.0 \pm 6.5 \mathrm{~mL} \mathrm{~g}^{-1}$ (Fig. 1c).

By week 7, the sludge biomass had entered Phase III of the aerobic granulation process. The mean particle size of the sludge biomass increased $90 \%$ from $193.0 \pm 16.7 \mu \mathrm{m}$ in week 6 to $367.0 \pm 68.1 \mu \mathrm{m}$ in week 7 (50th percentile) (Fig. 1b). The particle size and $\mathrm{SVI}_{5}$ of the sludge biomass continued to increase and decrease, respectively, over the remaining weeks. The MLSS of the sludge steadily increased from week 7 onwards (Fig. 1c). Over the entire 11 weeks, the reduction in settling time from 120 to 5 min was linked to the appearance of high density and compact sludge particles. This was associated with a mean particle size increase from $51.3 \pm 2.2$ to $792.4 \pm 130.6 \mu \mathrm{m}$ (Fig. 1b). Similarly, the $\mathrm{SVI}_{5}$ decreased significantly from $190.8 \pm 2.5$ to $16.0 \pm 2.1 \mathrm{~mL} \mathrm{~g}^{-1}$ (Fig. 1b). In addition, the MLSS of the sludge also increased from $3.9 \pm 0.5$ in week 6 to $12.7 \pm 0.6$ $\mathrm{mL} \mathrm{g}^{-1}$ by the end of week 11 . These observations indicated that the sludge biomass was mostly in granular form.

\section{Microbial community composition of floccular and granular sludge}

Here, the total genomic DNA of the granular sludge was sequenced to track the diversity and changes in

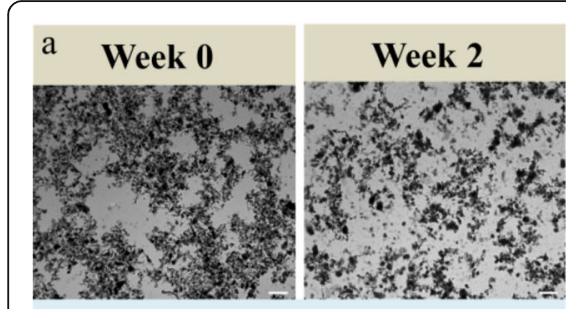

Phase I: Floccular

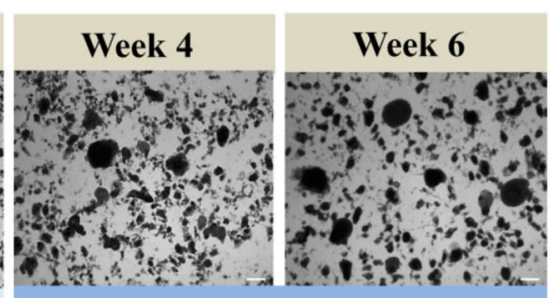

Phase II: Initiation

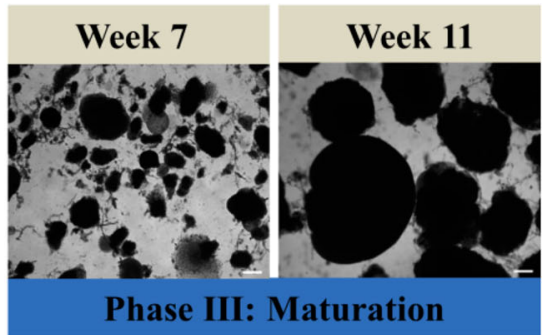

Phase III: Maturation
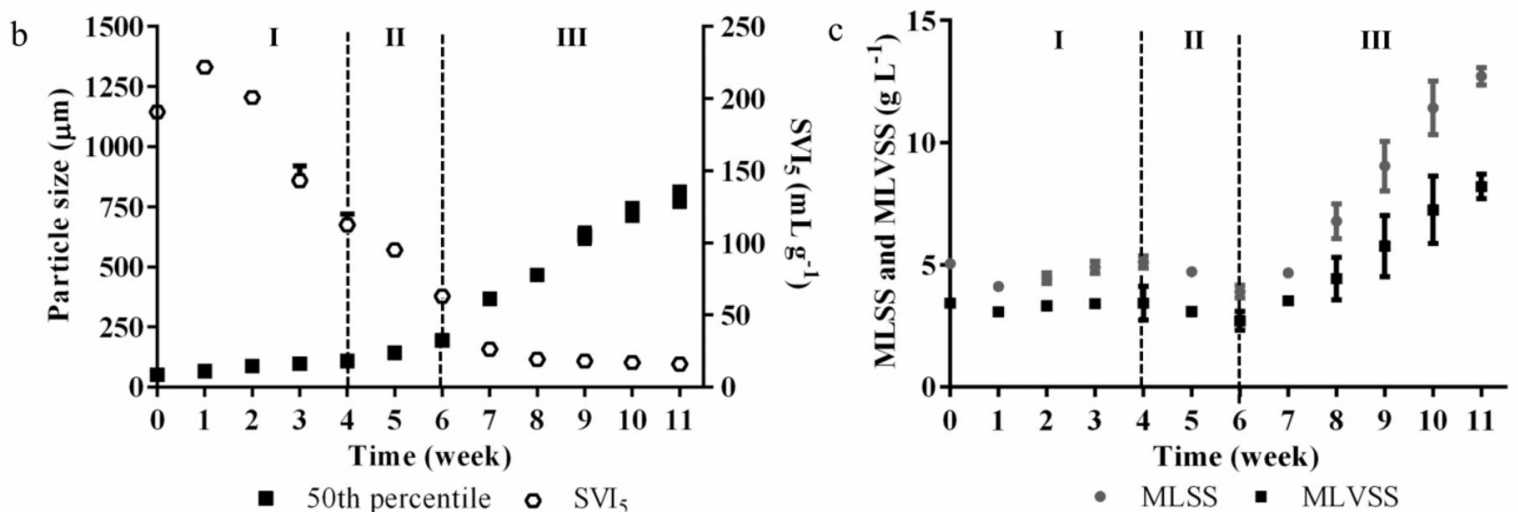

Fig. 1 Development of granules from floccular sludge. a Development of small granules from floccular sludge over 11 weeks based on

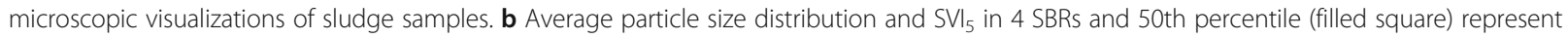
the percentage of total particles below the corresponding size distribution and the compactness of sludge particles as measured by $\mathrm{SV}_{5}$ (open circle), respectively. c Average sludge biomass concentrations represented by both MLSS (filled circle) and MLVSS (filled squares). Error bars represent standard deviations $(n=4)$. Magnification $\times 40($ Bar, $100 \mu \mathrm{m})$ 
bacteria abundance as granulation takes place over 11 weeks of reactor operation. Clustering based on the relative abundance of the microbial communities suggested that in the early floccular stages (weeks 0 and 1 ), the communities were similar across the 4 SBRs (Fig. S1a). However, from week 2, the communities between the reactors diverged, as reflected in changes in the community composition, as the reactors underwent granulation. Despite this, PERMANOVA showed that the reactors are not statistically different from each other $(P=0.184)$ (Table S1).

The genus 'Candidatus Accumulibacter', which is a polyphosphate accumulating organism (PAO) and nitrifier from the phylum Proteobacteria, was the most abundant, with an average increase from 3.6 to $63.53 \%$ by week 11 (Fig. 2). 'Candidatus Competibacter' and 'Candidatus Contendobacter', glycogen accumulating organisms (GAOs), did not change appreciably in abundance,

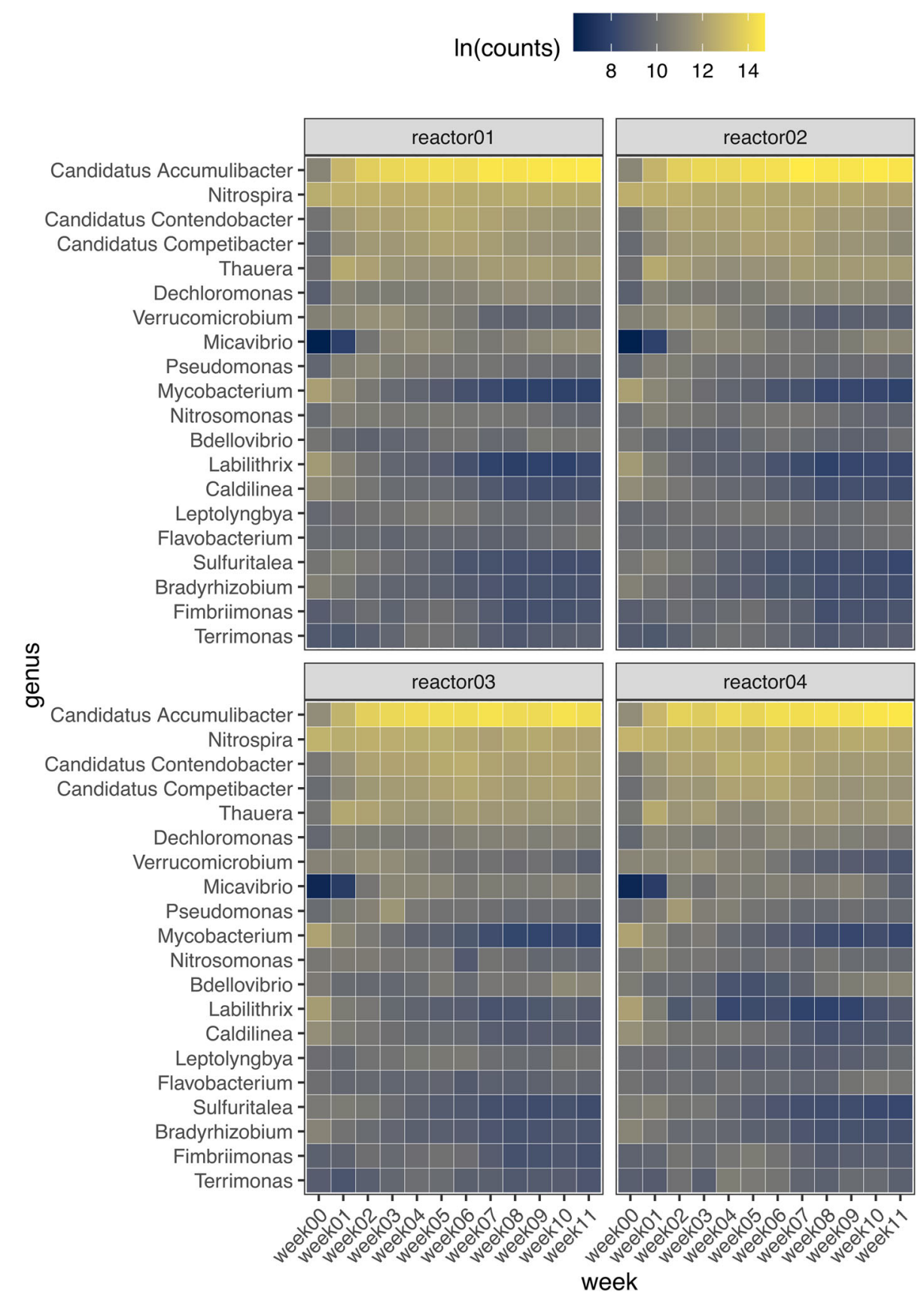

Fig. 2 Total abundance of bacterial populations in 4 SBRs over 11 weeks of granulation. The top 20 abundant bacterial genus based on metagenome (DNA) reads. All read counts are natural log transformed before analysis 
between 0.97 to $3.11 \%$ and 1.47 to $3.8 \%$, respectively (Fig. 2). Nitrifiers, such as Nitrospira, progressively decreased from 16.45 to $6.06 \%$ over the course of the experiment. There was a peak of Thauera (a denitrifier) at week 1 at $10.98 \%$ but reduced to $3.88 \%$ by the end of the experiment. The other members of the top 20 genera generally had a lower abundance with Terrimonas at the lowest between 0.3 and $0.97 \%$ (Fig. 2).
Bacteriophages exert a complex influence over their microbial hosts and additionally may play a structural component of the matrix [26-28]. Therefore, the relationship between granulation and bacteriophage community dynamics were also investigated here. Only DNA bacteriophages were targeted here and their sequences were assembled into viral contigs to study their relative abundance during granulation (Fig. 3a). Microviridae

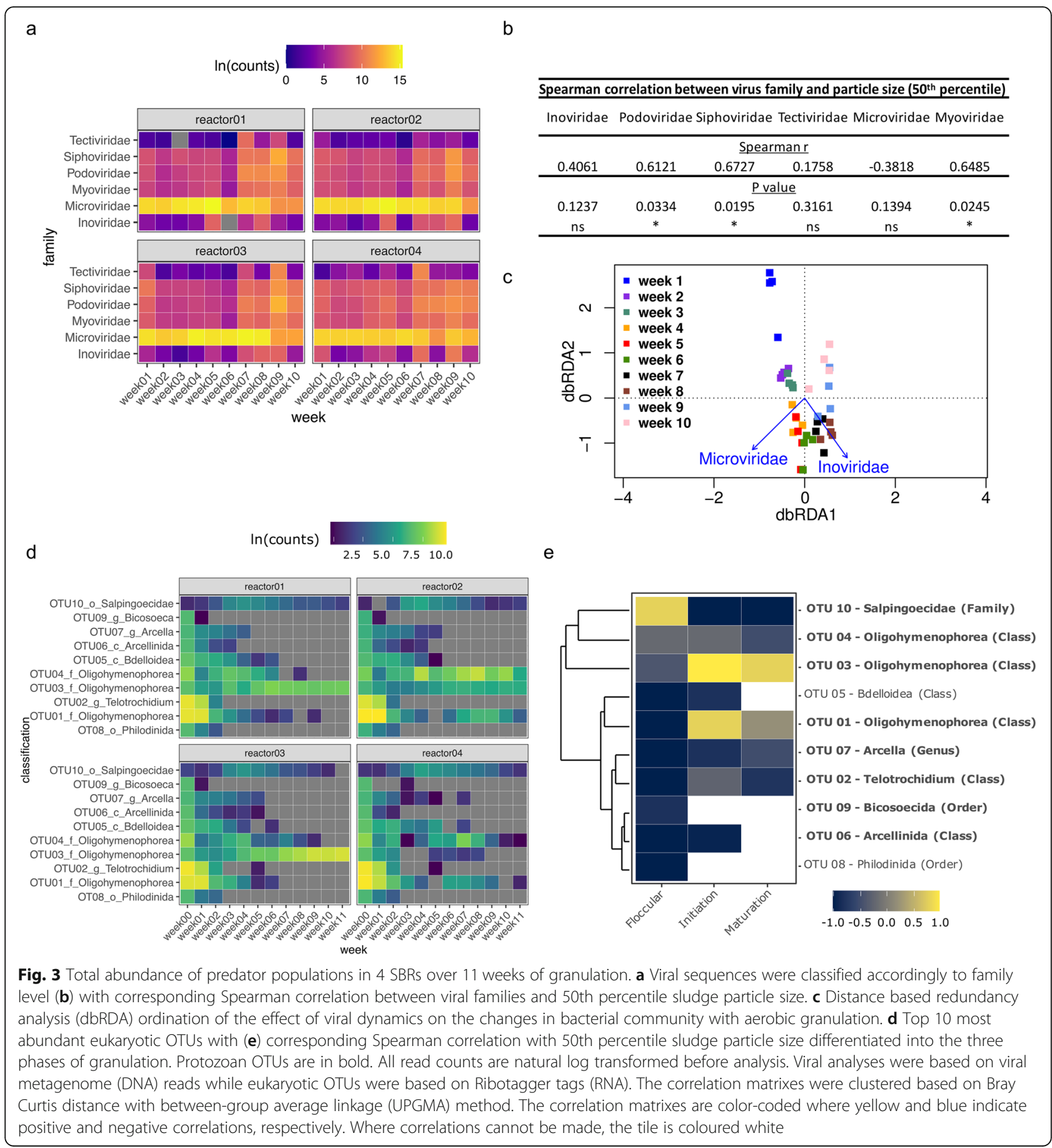


were the most abundant and present in all samples throughout reactor operation at 17 to $99.9 \%$. At the end of the initiation phase (week 7), Podoviridae and Siphoviridae began to significantly increase in abundance and at week 9, were the most abundant viral families after Microviridae at $11.15 \% \pm 1.66$ and $8.34 \% \pm 0.96 \%$, respectively. Inoviridae had an increase in abundance to $0.2 \% \pm 0.05 \%$ when the sludge developed into compact aggregates (week 5) and peaked at week 9 at $1.46 \% \pm$ $0.32 \%$ (Fig. 3a). There was a positive correlation between the increasing granule particle size and the viral counts of Siphoviridae, Microviridae and Myoviridae (Fig. 3b). Additionally, a distance based redundancy analysis (dbRDA) was performed to identify covariates which have an effect on the changes in bacterial community using viral family abundance [29]. This analysis suggested the Microviridae and Inoviridae viral families had an effect on the changes in bacterial community composition during the initiation phase (weeks 4 to 7 ) and maturation phase (weeks 8 to 9), respectively (Fig. 3c).

The effect of protozoan predation on aerobic granulation was investigated via total RNA sequencing as metagenomic sequencing did not yield sufficient reads for classification and annotation of eukaryotic sequences beyond the class level (Fig. S2). The abundance of the microbial populations was represented by the number of sequencing reads detected per OTU. Mean values were calculated for the number of sequences per OTU to represent the abundance in the four SBRs. A total of 10 OTUs represented approximately $95 \%$ of all sequencing reads. Within these 10 OTUs, there were 8 protozoan OTUs which were mostly represented by the genus Telotrochidium (OTU02), class Oligohymenophorea (OTU01, 03 and 04), genus Arcella and order Salpingoecidae (Fig. 3d).

The genus Telotrochidium is a group of free swimming peritrichous ciliates while the genus Arcella, and the family of Salpingoecidae represent testate amoebae and flagellates, respectively (Fig. 3d). The class Oligohymenophorea represents a large class of ciliated protozoa. Both OTU05 and 08 represented rotifers, which are metazoan predators of suspended microorganisms (Fig. 3d). During Phase I, the abundance of Telotrochidium (OTU02) decreased sharply by week 2 and was not detected in most reactors in the following weeks. The family Oligohymenophorea OTU01 also demonstrated gradual decline in abundance from week 0 to 3 . Both Oligohymenophorea (OTU 03 and 04) were constantly detected during Phase I in all reactors except in reactor 4 , where it was absent at week 03. Salpingoecidae (OTU10), of the flagellate family, was also constantly present from Phase I to III. However, as compact aggregates and granules formed by Phase II and III respectively, Oligohymenophorea (OTU03 and 04) were the most abundant eukaryotic members in the sludge biomass in all reactors. Testate amoeba, including OTU06 and OTU07, were not detected beyond week 5, by which time compact aggregates had formed.

Non-metric multi-dimensional scaling (nMDS) visualization of the eukaryotic communities during granulation demonstrated a high level of dissimilarity between the flocs at week 0 and granules at week 1 (Fig. S1c). Based on sludge particle size, the determinant of granulation, the majority of the eukaryotic OTUs, except for Salpingoecidae (OTU10), were positively correlated with the floccular particle size (Fig. 3e). In contrast, both Oligohymenophorea (OTU01 and 03) demonstrated a strong positive correlation with the particle size during the initiation and maturation phase. The remaining eukaryotic OTUs had a negative correlation during both the initiation and maturation phases (Fig. 3e).

A network analysis was undetaken to identify taxa that were possibly interacting with each other over the period of reactor operation (Fig. 4). There were 4 protozoan members that were correlated to the bacterial members of the reactor community. OTU01 and OTU07 were negatively correlated to 'Candidatus Competibacter' and Dechloromonas while OTU05 and OTU10 were positively correlated to Sulfuritalea and Mycobacterium, and Terrimonas, respectively. Most of the phages, except for Microviridae, were positively correlated to each other, with Podoviridae being the connecting node that is negatively correlated to Verrucomicrobium.

While sequencing provided insights into the eukaryotic communities in the sludge during granulation, microscopic observations were also performed to determine the presence of protozoa and other eukaryotes. Microscopic observations of sludge have also been utilized in membrane bioreactors to compliment sequencing data observations [30]. Swimming ciliates that were most likely Paramecium spp. were observed within the floccular sludge (Fig. 5a), while sessile ciliates were attached to the surfaces of the flocs (Fig. 5b). These ciliates represent the Oligohymenophorea OTUs detected by sequencing (Fig. 3d). Metazoans such as tardigardes (Fig. 5c) and rotifers (Fig. 5d) were frequently observed in the floccular sludge with crawling ciliates such as Aspidisca sp., circling the Phase I flocs (Fig. 5e). These rotifers were likely to be represented by OTU05 and 08 as identified in the sequencing data (Fig. 3d). These observations clearly indicated that the inoculum floccular sludge had a diverse community of protozoa present prior to seeding into the SBRs. Upon the formation of compact aggregates at Phase II, no swimming ciliates or large eukaryotes were observed, although rotifers were still occasionally present. Upon granule formation at Phase III, the frequency of crawling ciliates decreased significantly, while sessile ciliates were frequently 


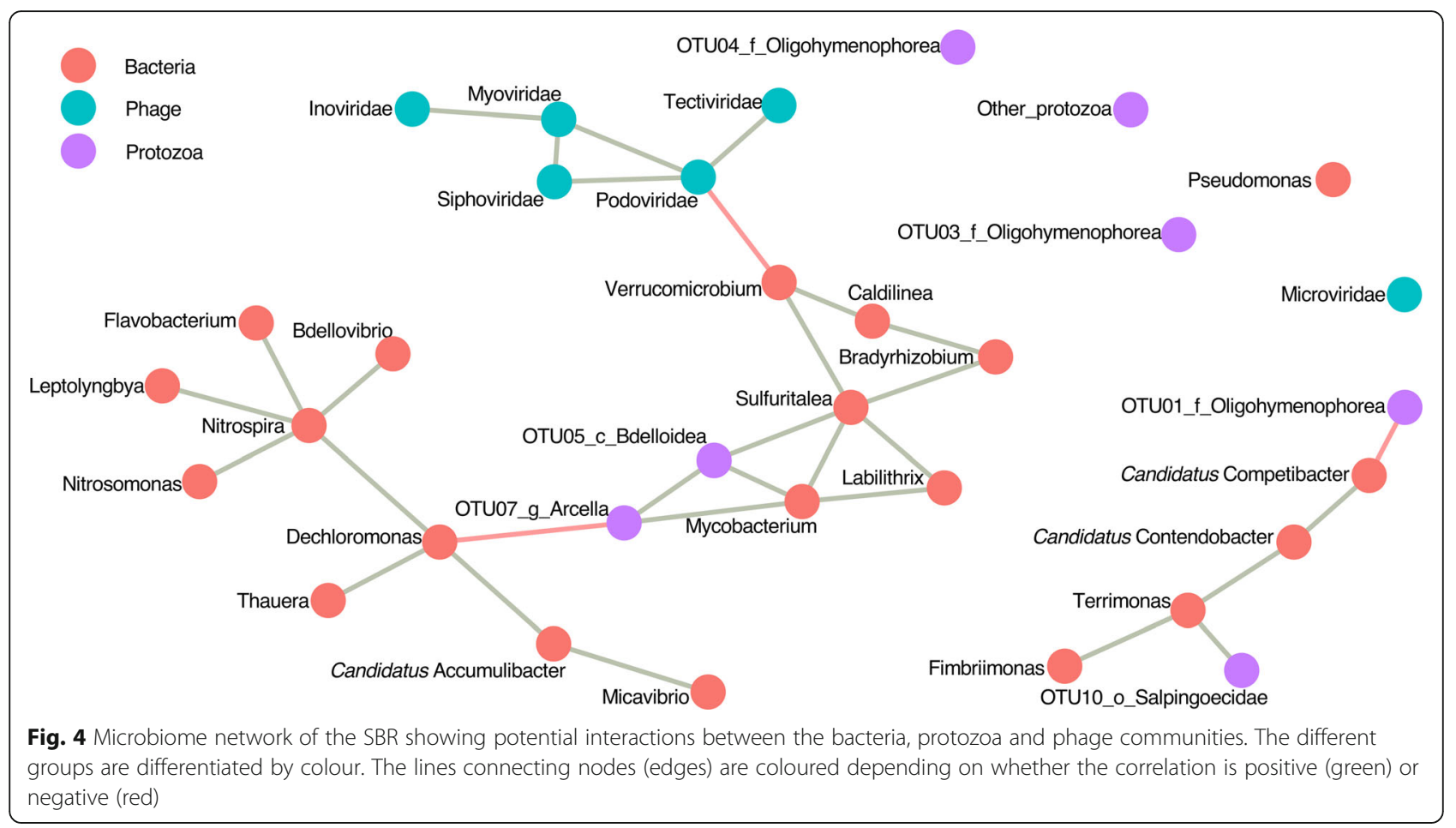

observed on the granule surfaces (Fig. $5 \mathrm{f}$ and g). The abundance of sessile ciliates, as determined by microscopy, were also reflected in the sequencing data where there were increases in Oligohymenophorea associated sequences (i.e OTU01, 03 and 04) in most reactors as granules formed.

\section{Development of aerobic granules from untreated and thiram treated floccular sludge}

Six mSBRs were seeded with activated floccular sludge and operated under conditions that were optimal for the aerobic granulation process over a period of 8 weeks. To investigate the role of protozoan predation in aerobic granulation, protozoa were removed from the floccular sludge by the addition of $20 \mathrm{mg} \mathrm{L}^{-1}$ thiram to the mSBRs and DMSO was added as a control. The concentration of thiram was previously optimized to minimize any negative effects on the viability of bacteria in the floccular sludge (data not shown). Microscopic observations of control floccular sludge indicated that the conversion of floccular into granular sludge began at week 4 (Fig. 6a). Compact aggregates were observed in the initiation phase and these aggregates continued to expand in size. The sludge entered the maturation phase at week 6 and remained in this phase until the end of the experiment at week 8 (Fig. 6a). In contrast, thiram treated sludge did not initiate granulation until week 6 and only started to mature by week 8 (Fig. 6a).

As the volumes of the mSBRs were too low to allow for particle sizing by the particle size analyser, particle sizing was obtained by quantitative image analysis. The initial mean sludge particle sizes were $84.36 \pm 12.41 \mu \mathrm{m}$ (Fig. 6b) and by week 2, the control sludge mean particle size was $89.61 \pm 5.94 \mu \mathrm{m}$, while the treated sludge was significantly smaller, $67.02 \pm 2.65 \mu \mathrm{m}$, than the control sludge (Fig. 6b). By week 7 , the treated sludge was $125.42 \pm 10.60 \mu \mathrm{m}$, which was similar to the control sludge particles, $122.71 \pm 23.00 \mu \mathrm{m}$ (Fig. 6b). By week 8 , there was a slight decrease in the control sludge $(104.60 \pm 17.57 \mu \mathrm{m})$, while the thiram treated sludge was significantly larger, $119.36 \mu \mathrm{m} \pm 6.05 \mu \mathrm{m}$ (Fig. 6b).

The $\mathrm{SVI}_{5}$ of the treated sludge was significantly higher than the control sludge from weeks 2 to 4 (Fig. 6c), suggesting that the thiram treated sludge was less dense and compact and hence required a longer settling time compared to the control sludge. However, from week 5 onwards, the $\mathrm{SVI}_{5}$ for the thiram treated sludge decreased and was not significantly different from the control sludge.

\section{Effects of thiram treatment on microbial communities during aerobic granulation}

The microbial communities in the two sludge types were compared by metacommunity sequencing of the V5 region of the 16S and 18S rRNA genes using the Ribotagger method [31]. A total of 30 OTUs, representing approximately $92 \%$ of the eukaryotic communities were selected for analysis. Within the inoculum sludge, the eukaryotic communities were dominated mainly by ciliated protozoa OTUs, e.g. OTUs 01, 02 and 03 (Fig. 7). 


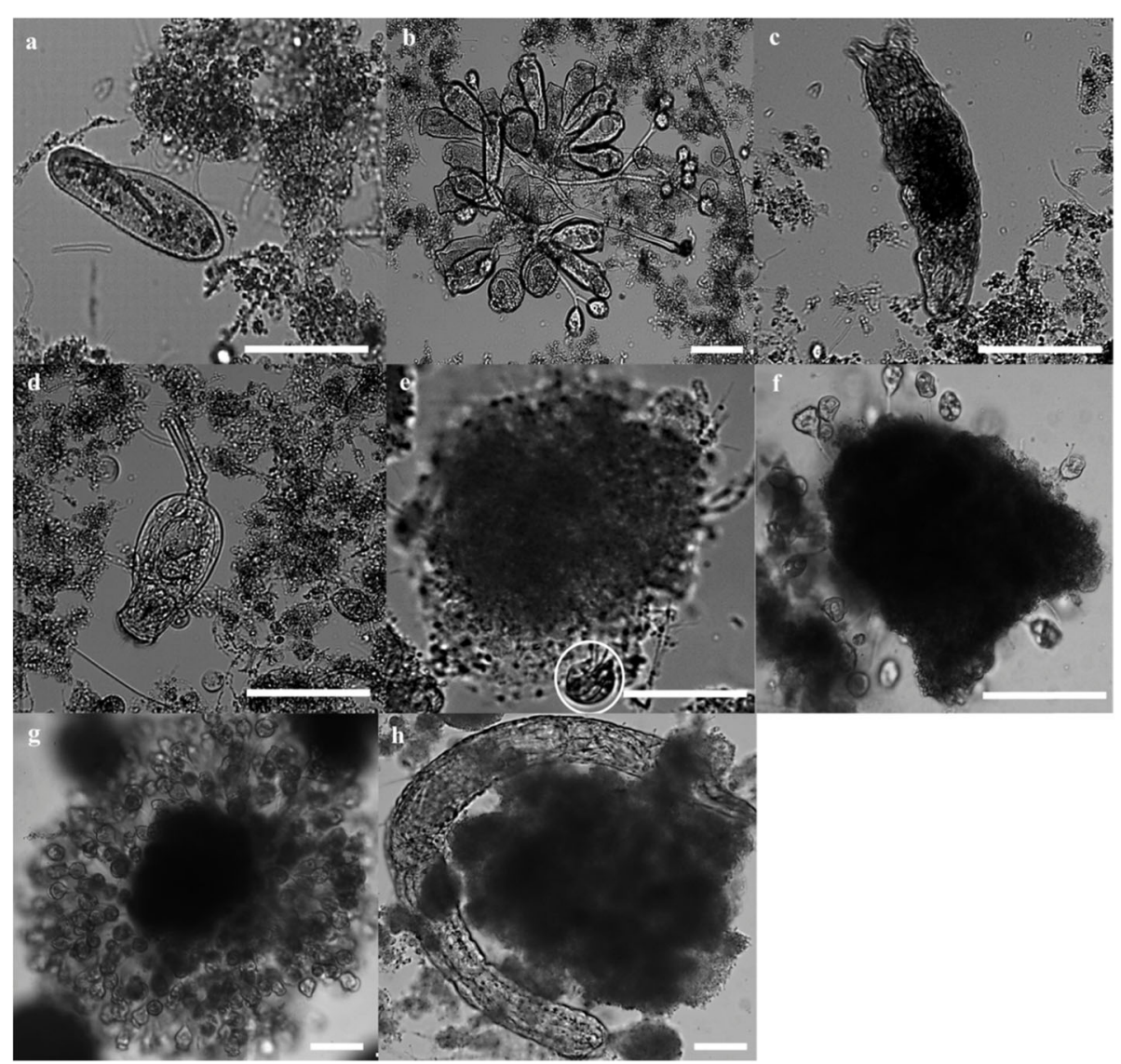

Fig. 5 Micrographs of protozoa and metazoa in floccular and granular sludge. a A swimming ciliate, Paramecium spp. and b sessile ciliates. c Metazoa such as tardigardes and $\mathbf{d}$ large rotifers from the genus Euchlanis. e Crawling ciliates (circled in white) were commonly sighted. $\mathbf{f}$ and $\mathbf{g}$ Sessile ciliates attached on the surface of granules. (Bar, $50 \mu \mathrm{m})$

As granulation progressed in the control mSBRs, the abundances of these OTUs were consistent, with Oligohymenophorea (OTU01) being the most dominant. Both Oligohymenophorea OTUs 02 and 07 showed a gradual decline in abundance while Oligohymenophorea (OTU26) was not detected beyond week 5. Swimming ciliates from the genus Paramecium (OTU 03) were not detected after week 1 (Fig. 7). In contrast to the swimming ciliates, crawling ciliates from the genus Aspidisca (OTU 23) were relatively abundant during granulation. However, these protozoan OTUs were mostly not detected after week 1 in the thiram treated sludge (Fig. 7). Interestingly, two flagellate associated OTUs, OTU08 and 24 , increased in abundance in the treated sludge from week 4 onwards.

Metazoan OTUs representing rotifers, e.g. OTUs 04, $05,06,15$ and 22, were also detected in relatively high abundance in the control sludge and were present throughout the entire granulation process (Fig. 7). In contrast, these rotifers were only detected at low abundance during the first 3 weeks in the treated sludge and were mostly not detected beyond week 4 . Eukaryotic communities in the control sludge did not changed drastically over time (Fig. S3a). However, eukaryotic communities in the thiram treated sludge diverged over time and were distinctly different from the control sludge from week 1 to 5 (Fig. S3a). This was likely due to the absence several dominant protozoan OTUs including OTU01, 02 and 07. Interestingly, the eukaryotic communities in the control and treated sludge began to converge from week 6 onwards, which was likely due to the resurgence of protozoan OTU08 and OTU24 (Fig. S3a).

Bacterial OTUs were also analyzed to determine if the absence of predators had any impact on the bacterial communities during aerobic granulation. Based on nMDS visualization, two distinct clusters were observed which indicated dissimilarities between the control and thiram treated bacterial communities during granulation (Fig. S3b). The bacterial communities remained relatively similar from week 2 to week 8 in the control sludge while the bacterial communities in the thiram treated sludge continue to change on a weekly basis (Fig. $\mathrm{S} 3 \mathrm{~b})$. In addition, there was no significant difference in the microbial communities between replicates of the 


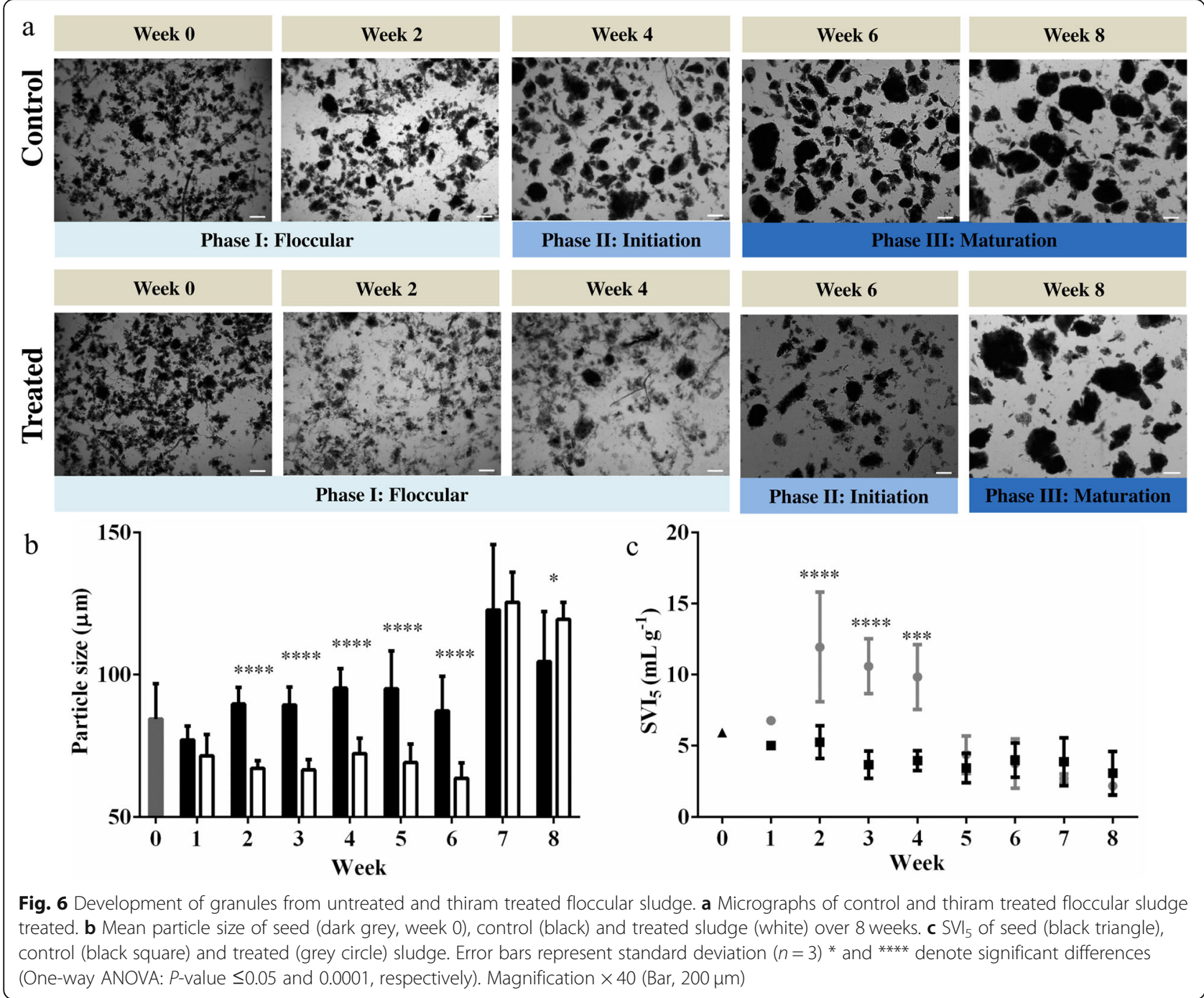

control or thiram sludge due to close clustering in each week (Fig. S3b).

In the control sludge, the bacterial communities were dominated mainly by PAOs such as 'Candidatus Accumulibacter' (OTU01), GAOs such as 'Candidatus Competibacter' (OTU04 and 05) and Nitrospira (OTU06) throughout 8 weeks of aerobic granulation (Fig. 8). The abundance of other bacterial members such as Zoogloea (OTU03), Thauera (OTU02), Dechloromonas (OTU07), 'Candidatus Competibacter' (OTU12, 17 and 19), 'Candidatus Contendobacter' (OTU09), Defluviicoccus (OTU18) and Actinobacteria (OTU20) remained relatively consistent during granulation (Fig. 8). In contrast, there was a decrease in the abundance of 'Candidatus Accumulibacter' (OTU01) and 'Candidatus Competibacter' (OTU04 and 05) from week 1 in the thiram treated sludge. The genus Nitrospira (OTU06) also demonstrated decline in abundance from week 1 onwards with no sign of recovery (Fig. 8). The decrease in abundance was also observed in 'Candidatus Competibacter' (OTU12, 17 and 19) and 'Candidatus Contendobacter' (OTU09) (Fig. 8). Interestingly, there were several bacterial OTUs such as Zoogloea (OTU03), Thauera (OTU02), Dechloromonas (OTU07) and Defluviicoccus (OTU18) that increased in abundance from week 1. However, as 'Candidatus Accumulibacter' (OTU01) began to gradually increase in abundance from week 5 , the abundance of Zoogloea (OTU03), Thauera (OTU02), Dechloromonas (OTU07) decreased. In contrast, OTU18 and 20 continued to gradually increase in abundance from week 5 onwards. 'Candidatus Accumulibacter' (OTU01) increased in abundance in the thiram treated sludge as it entered Phase II of granulation, where compact aggregates were formed.

\section{Discussion}

The abundance of 'Candidatus Accumulibacter' increased progressively during the granulation process 


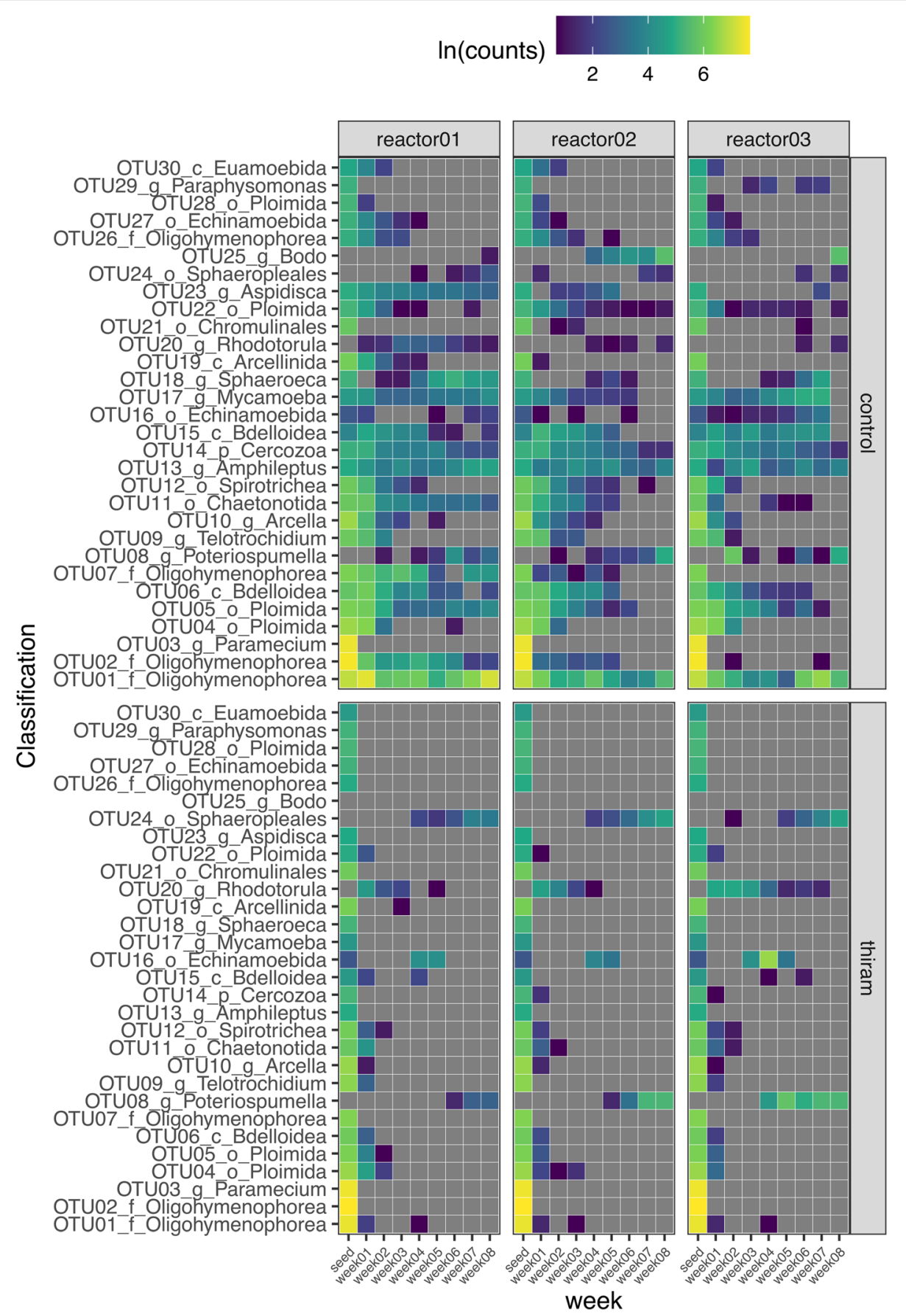

Fig. 7 Abundance of eukaryotic populations in control and treated mini-SBRs over 8 weeks of granulation. There were 3 control and 3 thiram treated mini-SBRs. The number of sequences per OTU in both control and treated were natural log transformed. These 30 OTUs represented approximately $92 \%$ of the total eukaryotic sequences

together with 'Candidatus Competibacter', 'Candidatus Contendobacter' and Nitrospira which were present at relatively high abundance (Fig. 2). Their higher abundance during granulation over other bacterial members suggests their close association with denser flocs or granules with better settling characteristics. This is similar to other biological nutrient removal systems where
'Candidatus Accumulibacter' and 'Candidatus Competibacter' were among the most abundant in the granular sludge community $[25,32,33]$. The higher abundance of 'Candidatus Accumulibacter' compared to 'Candidatus Competibacter' observed during the maturation phase could be partly due to the presence of propionate in the synthetic feed, which 'Candidatus Accumulibacter' has 


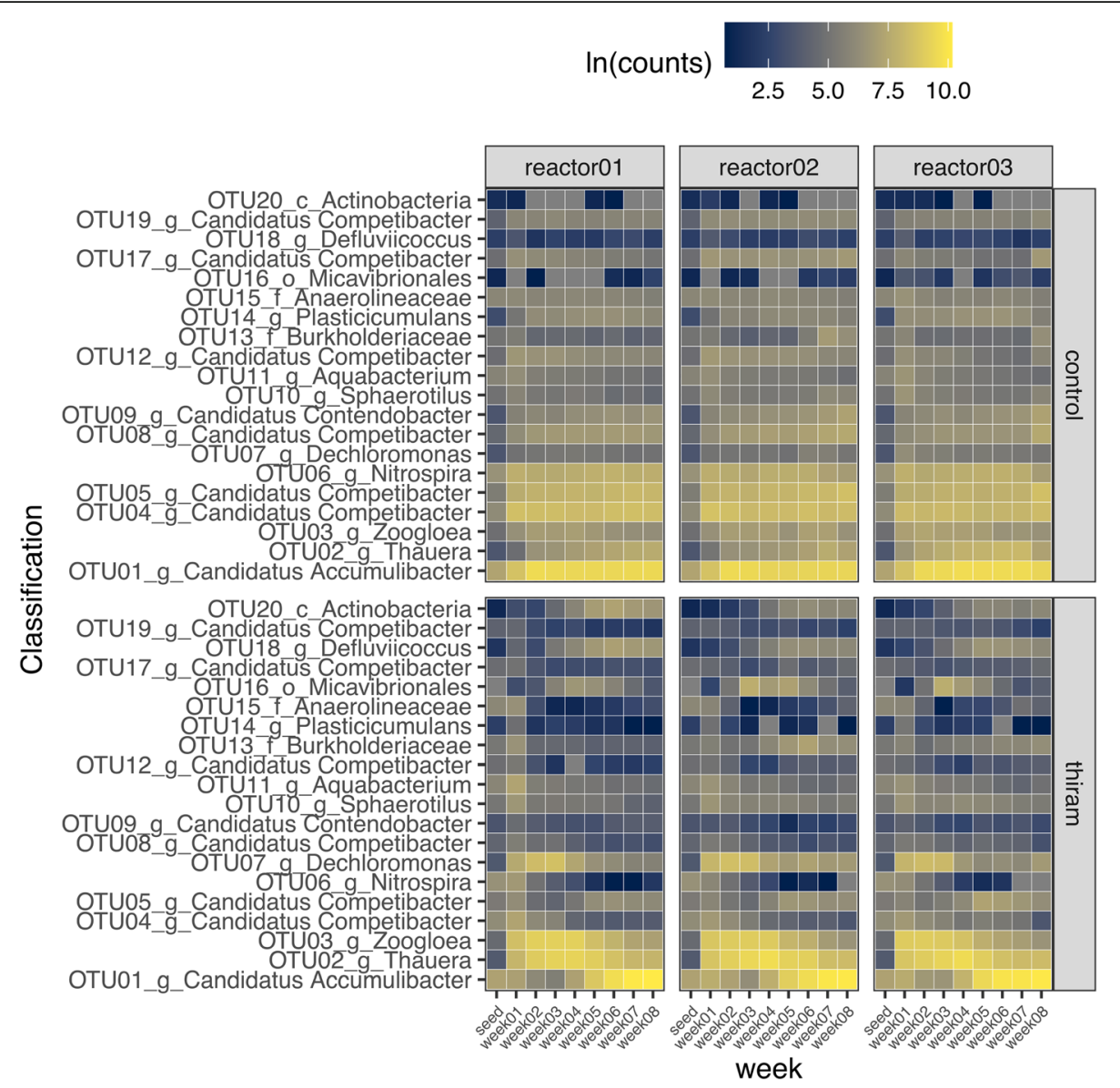

Fig. 8 Abundance of top 20 abundant bacterial OTUs in control and treated mini-SBRs over 8 weeks of granulation. There were 3 control and 3 thiram treated mini-SBRs. The number of sequences per OTU in both control and treated were natural log transformed before analysis

been shown to utilize/uptake more efficiently than 'Candidatus Competibacter' [34].

As 'Candidatus Accumulibacter' is often enriched for their capacity to remove phosphorus, their high abundance can increase their susceptibility to phage attack. It has been reported that abundant bacteriophage-like particles coupled with declining abundance of 'Candidatus Accumulibacter' and lysed 'Candidatus Accumulibacter' cells strongly suggested that phage infection was the main cause for 'Candidatus Accumulibacter' mortality [18]. Furthermore, the addition of bacteriophage-containing supernatant to other wastewater sludge also demonstrated similar decline in the abundance of 'Candidatus Accumulibacter', resulting in poor phosphorus removal [18]. These observations strongly suggest that phage infection can alter bacterial communities and their functionality of the systems in which they are present. The presence of certain bacteriophage families may be an indication that phage predation and the lysis of specific bacteria are required during the process of granulation. The change in abundance of Microviridae and Podoviridae (which are generally lytic phages) and Siphoviridae (temperate phages) were positively correlated with the increase in sludge particle size (Fig. 3b) and Microviridae were associated with changes in bacterial composition (Fig. 3c). While network analysis suggested that Podoviridae may be selectively predating on Verrucomicrobium (Fig. 4), the bacterial targets in flocs and granules of Microviridae, Podoviridae and Siphoviridae remain unclear. Verrucomicrobia are common in many wastewater treatment plants though a specific function has not been attributed to them $[35,36]$.

Although the role of lytic phages in the formation of aerobic granules is still unknown, a recent study demonstrated that bacteriophage-mediated extracellular DNA release was found to be vital for the structural stability of smaller aerobic granules [20]. Extracellular DNA containing clustered regularly interspaced short palindromic repeats (CRISPR) spacers was found to be part of the aerobic granular structure and extensive flocculation happened upon treatment with DNase I [20]. In our study here, the sequences associated with lytic phages from Microviridae and Podoviridae were constantly detected during the granulation process, suggesting that bacterial hosts associated with these families of lytic phages were present and phage lysis were actively 
ongoing throughout granulation. The increased abundance of Inoviridae (which are generally non-lytic filamentous bacteriophages) [37] in the reactor effluent at weeks 5, 7, 8 and 9 (Fig. 3a) coincided with the appearance of compact aggregates (Fig. 1). Inoviridae also seemed to influence changes in the bacterial community (Fig. 3c), in particular for weeks 4 to 8 , when the sludge was in the initiation to early maturation stages of granulation. Aerobic granule development has been suggested to be similar to biofilms that are surface associated [38], both containing EPS in their structure [26] as well as adhesion and cell-cell contact [39]. As for surfaceassociated biofilms, it is possible that filamentous phages play a role as a structural component in the granule as well. An example would be for the Pf4 filamentous phage in Pseudomonas aeruginosa biofilms where the phage organises the biofilm matrix into a liquid crystal structure that has increased viscosity and adhesion [27].

Other than bacteriophages, both microscopy and sequencing data analysis demonstrated an abundance of protozoa present during the floccular phase (Fig. $3 \mathrm{~d}$ and 4). Previous studies also reported a high abundance and diversity of protozoa in floccular sludge [40-42]. While protozoan predation in activated sludge has been suggested to facilitate increased biofilm production, a negative or lack of correlation was observed between the ciliated protozoa and particle sizes during the floccular phase. In contrast, there was a positive correlation between the flagellate family Salpingoecidae and the floccular particle size (Fig. 3e). Flagellates commonly predominate the activated sludge in the early stages as they consume lesser energy required for growth compared other larger protozoa such as ciliates. Interestingly, this family of flagellates was continuously detected throughout granulation and did not demonstrate any positive correlation with the particle sizes in the initiation or maturation phases. However, as compact aggregates formed and expanded during the initiation phase, sequences associated with Oligohymenophorea continued to be detected at relatively high abundance. While these sequences cannot clearly define the types of ciliated protozoa, microscopy analysis indicated that crawling and sessile ciliates increased in abundance. Grazing by crawling ciliates has been reported to stimulate the growth of microcolonies for surface attached biofilms [43]. In addition, activated sludge flocs that were cocultured with crawling ciliates from the genus Aspidisca demonstrated an increase in floc particle size and compactness [44]. Moreover, the motility of crawling ciliates such as Chilodonella can dislodge cells from biofilms $[43,45]$. These dislodged cells could then become a food source for the filter-feeding sessile ciliates. As more compact and dense granules formed, crawling ciliates are likely outcompeted by sessile ciliates whose growth is favored by an increase in granule surface area.

Sequencing analysis demonstrated ciliates from the class Oligohymenophorea were present throughout the granulation process (Fig. 3d). The class Oligohymenophorea consist of several subclasses such as Peritrichia that represent a distinctive group of sessile ciliates which are hypothesised to play an important role in granulation [22, 24, 46]. Sessile ciliates were often observed on the surfaces of activated sludge flocs and aerobic granules [21, 24, 47]. While sessile ciliates were observed microscopically on granules (Fig. $5 \mathrm{f}$ and g), there were no sequences from sessile ciliates that were classified to the genus level. Nonetheless, positive correlation with particle size during the initiation phase for the OTU 01 and 03 representing Oligohymenophorea suggests that these two classes of ciliated protozoa could play a more important role during the initiation phase of aerobic granulation rather than in the formation of mature granules. It is also possible that the change from flocs to granules acted as a form of selection pressure on the protozoan community, which also led to a significant reduction in the abundance and diversity of protozoa. For example, the presence of abundant, free-swimming protozoa will exert predation pressure on free swimming bacteria, which is known to promote biofilm formation such as compact aggregates and aerobic granules. However, the formation of biofilms simultaneously increases availability of substratum for colonization of sessile ciliates and surface grazing by crawling ciliates while reducing availability of free swimming bacteria for predatory flagellates and free swimming ciliates. Overall, the data suggest that the formation of aggregates favoured the growth of crawling and sessile ciliates while the compact and large granules favoured the colonisation of sessile ciliates.

Weber et al. [24] hypothesised that sessile ciliates could also act as nucleating agents for the attachment of bacteria. To investigate the role of protozoan predation in granulation, thiram was added into floccular sludge in this project. The resulting eukaryotic community in the thiram treated sludge was significantly different from the non-treated sludge where OTUs corresponding to protozoa were rarely detected by week 2 in the thiram treated sludge (Fig. 7). Importantly, there was very low or no detection of ciliated protozoa in the treated sludge. Without these ciliated protozoa particularly sessile ciliates, bacteria attachment was affected as the significant reduction of sessile agents contributed towards a loss of nucleating agents. It is likely that the absence of protozoa was responsible for the delayed increase in particle size. Protozoa have also been demonstrated to excrete growth stimulating products which could potentially induce flocculation [48]. For example, the co-incubation of activated sludge bacteria with sludge protozoa composed of attached, crawling ciliates, flagellates and amoeba for 
$48 \mathrm{~h}$, resulted in biofilms that had $2000 \%$ more biomass than the biofilms that were not exposed to protozoan predation [11]. In addition, polymeric substances such as extrusomes, cellular debris and undigested residues secreted from protozoa could also facilitate aggregation between bacterial flocs [49]. Hence, the absence of protozoan predation could have reduced the selection pressure aggregation, resulting in less dense flocs that settle poorly. Poor settling of the treated sludge could also have resulted in larger losses of sludge biomass during discharge. This finding corresponded to previous observations where ciliated protozoa were important for the formation of compact aggregates which leads to granule formation.

In the absence of protozoan predators, the floccular sludge demonstrated poor compactness and settling. Both 'Candidatus Accumulibacter' and 'Candidatus Competibacter' were replaced by the genera Thauera and Zoogloea as the dominant members of the bacterial community in the treated floccular sludge in Phase I. The 'Candidatus Accumulibacter' could have also been replaced by both genera of Thauera and Dechloromonas, which are PAOs that are also capable of denitrification [50]. The proliferation of Zoogloea was likely due to insufficient retention of sludge [32, 51]. Zoogloea are flocforming bacteria that produce aggregates enveloped in gelatinous matrixes that could have initiated the formation of compact aggregates. However, the initiation phase was delayed in the thiram treated reactors despite the abundance of Zoogloea associated sequences and it is possible that the delay in initiation was due to the absence of protozoa.

While there was a delay in initiation, the thiram treated sludge ultimately granulated by week 8 and we hypothesised that it could be largely attributed to the emergence of 'Candidatus Accumulibacter' after week 5. In contrast, the control sludge, which was dominated mainly by 'Candidatus Accumulibacter' and 'Candidatus Competibacter', entered the initiation and granulation phase earlier at weeks 4 and 6, respectively. These observations further support that the high abundance of 'Candidatus Accumulibacter' and 'Candidatus Competibacter' are strong potential contributors towards the formation of aerobic granules. Other than bacteria, it is also likely that protozoan predation plays a partial role in enhancing the formation of aerobic granules by reducing the time to initiate compact aggregates formation.

The limitations of this study are that while the mechanistic link between protozoan predation and granulation was directly tested, the effect of bacteriophages on granulation is correlative. In addition, the current sequencing data is unable to define Oligohymenophorea sequences to the genus or species level. Hence, any true impact on granulation from these ciliates will require isolation of specific protozoa and subsequently adding them back in significant amounts in floccular or granular sludge. Future work should address these issues by isolating phages and protozoa from key points in the granular development (e.g. initiation phase) and adding back these phages to either floccular or granular sludge to see their more direct impacts on granulation.

\section{Conclusions}

Predation by bacteriophages and protozoa can influence the diversity and structure of the bacterial community. The alteration of bacterial community composition subsequently affected the rate of granulation of floccular sludge. While physical parameters such as settling time have significant effects on promoting granulation, we have also demonstrated the potential role of bacteriophage and protozoa in promoting granulation through physical means such as bacterial attachment on phage filaments or sessile ciliates.

\section{Methods}

Sequencing batch reactor setup and operation

To characterize the protozoan communities during aerobic granulation, four independent SBRs were seeded with activated floccular sludge from the Ulu Pandan Wastewater Treatment Plant, Singapore, as previously described [52, 53]. Briefly, each SBR had a final working volume of $2 \mathrm{~L}$ and was operated in a $6 \mathrm{~h}$ cycle comprising two different phases: Phase I - feeding $(8 \mathrm{~min})$, anaerobic $(60 \mathrm{~min})$, aerobic $(80 \mathrm{~min}$ at day 0 , with a gradual increase to $95 \mathrm{~min}$ by week 5 ) and anoxic (40 $\mathrm{min}$ at day 0 , with a gradual increase to $50 \mathrm{~min}$ by week 5); Phase II - feeding ( $2 \mathrm{~min}$ ), anaerobic (30 $\mathrm{min})$, aerobic (40 min at day 0 and gradual increase to $70 \mathrm{~min}$ by week $5)$ and anoxic (30 min). Each cycle was completed with a settling stage $(120 \mathrm{~min}$ at day 0 , with a gradual decrease to $5 \mathrm{~min}$ by the end of week 6 ) and a $10 \mathrm{~min}$ decanting stage. The settling time was maintained at $5 \mathrm{~min}$ per cycle from week 6 onwards.

A total volume of $1 \mathrm{~L}$ of synthetic wastewater was supplied to each SBR by Phase II and $1 \mathrm{~L}$ of effluent was discharged at the completion of each cycle. Synthetic wastewater was prepared as previously described [54, 55]. Dissolved oxygen (DO) levels were maintained at $0.0 \mathrm{mg} \mathrm{L}^{-1}$ during anaerobic phases via intermittent nitrogen sparging and maintained between 3.0 to $4.0 \mathrm{mg}$ $\mathrm{L}^{-1}$ during aerobic phases by compressed air sparging. Sparging of both nitrogen and air provided complete mixing of the sludge and the hydrodynamic shear force required for aerobic granulation. The $\mathrm{pH}$ of each SBR was maintained between 6.8 and 8.2 by dosing with 0.1 $\mathrm{M} \mathrm{HCl}$ and $0.1 \mathrm{M} \mathrm{NaOH}$ as required. Both $\mathrm{pH}$ and $\mathrm{DO}$ levels were monitored by inline probes connected to a programmable logic controller (PLC). 
Mixed liquor suspended solids (MLSS) and mixed liquor volatile suspended solids (MLVSS), were determined using APHA standard engineering methods [56]. Sludge density and compactness was measured by sludge volumetric index at $5 \mathrm{~min}\left(\mathrm{SVI}_{5}\right)$ as described [7]. Sludge particle sizes were determined using a laser diffraction particle size analyser (SALD-3101, Shimadzu, Japan) and their morphology was recorded by light microscopy (Primo Star, Carl Zeiss, Germany). At the end of each cycle study, well-mixed sludge samples of $1 \mathrm{~mL}$ were collected from each reactor at the end of Phase II anoxic stage. These sludge samples were centrifuged at $8000 \mathrm{~g}$ for $5 \mathrm{~min}$ and snap frozen in liquid nitrogen prior to storage at $-80^{\circ} \mathrm{C}$.

\section{Total genomic DNA extraction from aerobic granular sludge}

One milliliter of suspended sludge was pelleted by centrifugation for $10 \mathrm{~min}$ at $10,000 \mathrm{~g}$. The total genomic DNA was extracted using the sludge pellet with the FastDNA $^{\text {ma }}$ SPIN Kit for Soil (MP Biomedical, USA) mostly according to the manufacturer's guidelines. Homogenization was performed twice in the FastPrep Instrument for $40 \mathrm{~s}$ at a speed setting of 6.0. The extracted genomic DNA was then cleaned up using the Genomic DNA Clean \& Concentrator (Zymo Research, USA) according to the manufacturer's guidelines. The concentration of the DNA was quantified using the dsDNA HS Assay Kit and the Qubit $^{\circ}$ 2.0 Fluorometer (Life Technologies, USA) before sequencing on the Illumina $\mathrm{HiSeq}$ as $250 \mathrm{bp}$ paired end reads.

\section{Total metagenome analysis of the aerobic granular sludge}

The quality of the metagenomic reads was assessed using FastQC ( $\mathrm{v}$ 0.11.5) before it was adapter and quality trimmed using BBMap (v 36.38) [57]. Contigs were co-assembled using MEGAHIT (v 1.0.6-3) [58] with the meta-sensitive preset mode before ORF prediction was done using the meta mode of Prodigal ( $\mathrm{v}$ 2.6.3) [59]. Using nucleic acid ORF sequences, redundancy was removed using cd-hit-est (v 4.6.8) [60] with the options for $95 \%$ sequence identity and word length of 10. The non-redundant ORF sequences were then used in a protein homology search using the Blastx function of DIAMOND ( $v$ 0.8.22) [61] against the NCBI nr database. Based on the Blastx output, the lowest common ancestor (LCA) annotation for the contigs were performed using MEGAN6 Community Edition ( $v$ 6.8.12) [62]. To obtain the contig abundance table, the metagenome reads were mapped to the co-assembled contigs using Bowtie2 ( $\mathrm{v}$ 2.2.6) [63] before read coverage was obtained with the idxstats function of Samtools (v 1.3.1) [64]. The contig abundance and LCA-annotated contigs were then analysed using Phyloseq (v 1.22.3) [65] in R.

\section{Viral fraction sampling and concentration}

During SBR operation, the viral fraction was collected and concentrated as previously described [52]. Briefly, effluent from each reactor was discharged into their respective containers before transferring into $25 \mathrm{~L}$ carboys. Samples were collected from weeks 1 to 10 of the study. The initial filtrate was obtained by passing through a $25 \mu \mathrm{m}$ filter bag (Puridea, Singapore) to remove any suspended biomass before storing at $4{ }^{\circ} \mathrm{C}$. The filtrate was pooled over $4 \mathrm{~d}$ to obtain $20 \mathrm{~L}$, at which time, $2 \mathrm{~mL}$ of DNase I (200 U/mL) (Calbiochem, USA) was added to the samples to digest any extracellular DNA. Bacteria were then removed by passing the filtered effluent through a $0.2 \mu \mathrm{m}$ Sartocon Slice Disposable tangential flow filter (TFF) (Sartorius Stedim, Germany). To concentrate the viral fraction, the permeate was concentrated using a $100 \mathrm{kDa}$ Sartocon Slice Dispostable TFF (Sartorius Stedim, Germany). In this process, the fluid phase and particles smaller than $100 \mathrm{kDa}$ were removed while the viral fraction remained in the reservoir. To elute the viral fraction, SM buffer $(100 \mathrm{mM} \mathrm{NaCl}, 8 \mathrm{mM}$ $\mathrm{MgSO}_{4}, 50 \mathrm{mM}$ Tris-Cl at $\left.\mathrm{pH} 7.5\right)$ was added to the phage reservoir until the volume was reduced to a 100 $\mathrm{mL}$. The viral fraction was further concentrated using the Vivaspin 20 100,000 MWCO Centrifugal Concentrators (Sartorius Stedium, Germany) by centrifuging at $5000 \mathrm{~g}$ for $30 \mathrm{~min}$ at $4{ }^{\circ} \mathrm{C}$ until a volume between 2 to 3 $\mathrm{mL}$ was obtained and stored at $-80^{\circ} \mathrm{C}$ as $200 \mu \mathrm{L}$ aliquots.

\section{Bacteriophage nucleic acid extraction and multiple displacement amplification}

Nucleic acids were extracted using the QIAamp MinElute Virus Spin kit (Qiagen, Germany) from $200 \mu \mathrm{L}$ of concentrated viral fraction according to manufacturer's guidelines. The viral DNA was used for whole genome multiple displacement amplification (MDA) using random hexamers with the illustra GenomiPhi V2 DNA amplification kit (Cytiva, USA) according to the manufacturer's guidelines. The amplified DNA was then purified using the ethanol precipitation method [66]. Briefly, sodium acetate was added and mixed to the amplified sample to a final concentration of $0.3 \mathrm{M}$ at pH 5.2. Two volumes of cold $100 \%$ molecular grade ethanol was added and incubated overnight at $-20^{\circ} \mathrm{C}$. After incubation, the sample was centrifuged at $15,000 \mathrm{~g}$ for $30 \mathrm{~min}$ and the supernatant was removed. One $\mathrm{mL}$ of $70 \%$ ethanol was then added and incubated at $-20^{\circ} \mathrm{C}$ for $2 \mathrm{~h}$ before centrifuging at $15,000 \mathrm{~g}$ for $30 \mathrm{~min}$ to pellet DNA. The supernatant was discarded and the pellet air dried for 5 min before resuspension in sterile $\mathrm{dH}_{2} \mathrm{O}$. 


\section{Analysis of the viral fraction}

The quality of the metavirome reads was assessed using FastQC (v 0.11.5) before adapter and quality trimming using BBMap (v 36.38) [57]. Contig co-assembly was done using the MEGAHIT ( $\mathrm{v}$ 1.0.6-3) meta-sensitive preset mode [58] before doing ORF prediction using the Prodigal ( $v$ 2.6.3) meta mode [59]. The viral ORF sequences were used in a protein homology search using the DIAMOND (v 0.8.22) Blastp program against the A CLAssification of Mobile genetic Elements (ACLAME) database. Based on the output, the LCA annotation for the viral contigs were obtained using MEGAN6 Community Edition ( $\mathrm{v}$ 6.8.12) [62]. The metavirome reads were then mapped to the viral contigs using Bowtie $2(\mathrm{v}$ 2.2.6) [63] before obtaining read coverage using the idxstats function of Samtools (v 1.3.1) [64]. The LCA annotated viral contigs and their abundances were then used for downstream analyses.

\section{Mini-sequencing batch reactors setup and operation} Mini-SBRs (mSBR) were seeded with activated floccular sludge from the Ulu Pandan Wastewater Treatment Plant, Singapore. For floccular sludge experiments, both controls and treatments were performed in triplicate while granular sludge experiments were performed in duplicate. Each $\mathrm{mSBR}$ had a final working volume of 30 $\mathrm{mL}$ and was operated in a $6 \mathrm{~h}$ cycle: feeding $(10 \mathrm{~min})$, anaerobic $(100 \mathrm{~min})$, aerobic $(110 \mathrm{~min}$ at day 0 , with a gradual increase to $120 \mathrm{~min}$ by the end of week 1) and anoxic (100 min) phases. Each cycle was completed with a settling stage (30 min at day 0 , with a gradual decrease to $20 \mathrm{~min}$ by the end of week 1 ) and a 10 min decanting stage. The settling time was maintained at $20 \mathrm{~min}$ per cycle from the end of week 1 onwards.

Synthetic wastewater $(15 \mathrm{~mL})$ was fed to each $\mathrm{mSBR}$ in Phase II and $15 \mathrm{~mL}$ of treated effluent was discharged at the end of the cycle. For the inhibition of eukaryotes, thiram (Sigma Aldrich, Germany) was dissolved in dimethyl sulfoxide (DMSO) to obtain a stock solution of $20 \mathrm{gL}^{-1}$ for treatment of the floccular sludge. Thiram has been shown to inhibit protozoa with minimal impacts on bacterial activities [67]. Based on optimization studies, thiram was added to each reactor once per day after feeding to obtain a final concentration of $20 \mathrm{mg}$ $\mathrm{L}^{-1}$ (data not shown), while DMSO was added to control mSBRs. Both DMSO and thiram treatment of sludge was completed by week 2 . Both control and treated mSBRs were operated from weeks 3 to 8 without the addition of DMSO or thiram. To achieve anaerobic and aerobic conditions, nitrogen and compressed air were sparged intermittently into the mSBRs.

The average particle diameter of the floccular sludge was determined by analyzing images of sludge, taken in triplicate for each $\mathrm{mSBR}$, on a weekly basis using ImageJ
(National Institute of Health, USA). For enumeration of protozoa, triplicate $10 \mu \mathrm{L}$ aliquots were removed from each $\mathrm{mSBR}$ and the numbers of protozoa determined using light microscopy (Primo Star, Carl Zeiss, Germany). Samples $(1 \mathrm{~mL})$ were collected from each $\mathrm{mSBR}$ at the end of Phase II anoxic stage, centrifuged at $8000 \mathrm{~g}$ for $5 \mathrm{~min}$ and snap frozen in liquid nitrogen prior to storage at $-80^{\circ} \mathrm{C}$.

\section{RNA extractions for total RNA sequencing and analysis}

Total RNA was extracted from sludge samples using the Soil, Fecal and Plant RNA kit (Zymo Research, USA) as described [68, 69], according to the manufacturer's guidelines. Extracted RNA underwent a single round of DNase treatment to remove residual DNA (TURBO ${ }^{\mathrm{ms}}$ DNase kit; Invitrogen, Singapore). The quality of the extracted RNA was measured by spectrophotometry (Nanodrop; Thermo Scientific, USA). The concentration of RNA and residual DNA was determined by fluorometry (Qubit ${ }^{\circ}$ 2.0 Fluorometer; Invitrogen, USA), using the Qubit ${ }^{\circ}$ RNA broad range assay kit (Invitrogen, USA) and Qubit ${ }^{\circ}$ DNA high sensitivity range assay kit respectively, following the manufacturer's guidelines. In addition, the integrity of the RNA was determined using the RNA Analysis ScreenTape and 2200 Tapestation instrument (Agilent Technologies, Singapore) and reported as the RNA Integrity Number (RIN). These RNA samples were subsequently sent for RNA library preparation prior to pooling and sequencing on an Illumina HiSeq 2500 System (Illumina Inc.) using $100 \mathrm{bp}$ pairedend (PE) sequencing as per the manufacturer's guidelines.

\section{Total RNA sequencing and analysis}

The microbial composition of the floccular and granular sludge was determined by analysis of the sequence data using the Ribotagger fast tag-based approach [31]. Briefly, universal recognition profiles that target bacteria, Archaea and eukaryotes were selected for each of the hypervariable regions of both $16 \mathrm{~S}$ and $18 \mathrm{~S}$ rRNA (e.g. V4, V5, V6 and V7) (Xie et al. [31]). These universal recognition profiles were used to scan the sequencing reads to obtain 33 nucleotides (nt) downstream of the primers (Xie et al. [31]). Each of these $33 \mathrm{nt}$ tags were defined as a ribotag and each ribotag was screened against the SILVA database to map it to a known organism. Hence, each ribotag was used as a signature sequence to represent one operational taxonomic unit (OTU). Here, only the sequencing reads from the V5 regions of $18 \mathrm{~S}$ rRNA were used to represent the abundance of protozoan communities. Based on the lowest number of total sequencing reads within the samples set, these V5 sequencing reads were randomly subsampled based 
on a seed value of 100 using the seqtk FASTQ program (https://github.com/lh3/seqtk).

\section{Network analysis}

The combined abundance table of the bacterial, protozoan and viral communities were combined before it was loaded as a phyloseq object using Phyloseq ( $\mathrm{v}$ 1.22.3) [65] in R. The network analysis was performed using the SpiecEasi package (v 1.1.0) [70]. Briefly, the abundance table was normalised using centered log-ratio transformation before inverse covariance estimation was done. The stability of the network was inferred using the package's Stability Approach to Regularization Selection (StARS) criterion. The following parameters were used: method $=$ "mb", lambda $\cdot$ min. ratio $=0.05$, nlambda $=100$.

\section{Statistical analysis}

Correlation studies for protozoa and bacteriophages were performed by calculating Spearman correlation coefficient using Prism (Graphpad 6.0). The resulting protozoa matrices were clustered hierarchically based firstly by obtaining the Bray- Curtis dissimilarity matrix and then clustering using the hclust function in vegan (v.5-6) [71] in R. The distance matrices were used for non-metric multi-dimensional scaling (NMDS) to determine the level of similarity or dissimilarity between of samples based no bacteria and eukaryotic communities.

\section{Supplementary Information}

The online version contains supplementary material available at https://doi. org/10.1186/s12866-021-02156-8

\section{Additional file 1}

\section{Acknowledgements}

Not applicable.

\begin{abstract}
Authors' contributions
SHC, MHI, CHT and SAR conceived and designed the experimental studies. SHC, MHI and CHT performed most of the experiments. SHC and MHI analysed most of the experiments. SAR and DM edited the paper. All authors had substantial contributions to the paper, were involved in writing, approved the final version, and are accountable for all aspects of the work.
\end{abstract}

\section{Funding}

This research was supported by the Singapore Centre for Environmental Life Sciences Engineering (SCELSE), whose research is supported by the National Research Foundation Singapore, Ministry of Education, Nanyang Technological University and National University of Singapore, under its Research Centre of Excellence Programme. MH Ismail was supported by the National Research Foundation Singapore under its National Research Foundation (NRF) Environmental and Water Technologies (EWT) PhD Scholarship Programme, administered by the Environment and Water Industry Programme Office (EWI).

\section{Availability of data and materials}

The datasets generated and/or analysed during the current study are available in the DR-NTU (Data) repository, https://doi.org/10.21979/N9/ TBOIOY.

\section{Declarations}

Ethics approval and consent to participate

Not applicable.

\section{Consent for publication}

Not applicable.

\section{Competing interests}

The authors declare that they have no competing interests.

\section{Author details}

${ }^{1}$ Singapore Centre for Environmental Life Sciences Engineering, Nanyang Technological University, 60 Nanyang Drive, Singapore 637551, Singapore. ${ }^{2}$ Interdisciplinary Graduate School, Nanyang Technological University, Singapore, Singapore. ${ }^{3}$ School of Biological Sciences, Nanyang Technological University, Singapore, Singapore. ${ }^{4}$ School of Materials Science and Engineering, Nanyang Technological University, Singapore, Singapore. ${ }^{5}$ The iThree Institute, University of Technology Sydney, Sydney, Australia.

Received: 13 January 2021 Accepted: 8 March 2021

Published online: 27 March 2021

\section{References}

1. Beun JJ, Hendriks A, Van Loosdrecht MCM, Morgenroth E, Wilderer PA, Heijnen JJ. Aerobic granulation in a sequencing batch reactor. Water Res. 1999;33(10):2283-90. https://doi.org/10.1016/S0043-1354(98)00463-1.

2. Morgenroth E, Sherden T, van Loosdrecht MCM, Heijnen JJ, Wilderer PA. Aerobic granular sludge in a sequencing batch reactor. Water Res. 1997; 31(12):3191-4. https://doi.org/10.1016/S0043-1354(97)00216-9.

3. McSwain BS, Irvine RL, Wilderer PA. The influence of settling time on the formation of aerobic granules. Water Sci Technol. 2004;50(10):195-202. https://doi.org/10.2166/wst.2004.0643.

4. Qin L, Liu Y, Tay J-H. Effect of settling time on aerobic granulation in sequencing batch reactor. Biochem Eng J. 2004;21(1):47-52. https://doi. org/10.1016/j.bej.2004.03.005

5. Tay JH, Liu QS, Liu Y. The effects of shear force on the formation, structure and metabolism of aerobic granules. Appl Microbiol Biotechnol. 2001;57(1): 227-33. https://doi.org/10.1007/s002530100766.

6. Winkler MKH, Kleerebezem R, Khunjar WO, de Bruin B, van Loosdrecht MCM Evaluating the solid retention time of bacteria in flocculent and granular sludge. Water Res. 2012;46(16):4973-80. https://doi.org/10.1016/j.watres.2 012.06.027.

7. Liu Y. Wastewater purification: Aerobic granulation in sequencing batch reactors. Boca Raton: Taylor \& Francis; 2008.

8. Tan CH, Koh KS, Xie C, Tay M, Zhou Y, Williams R, Ng WJ, Rice SA, Kjelleberg $S$. The role of quorum sensing signalling in EPS production and the assembly of a sludge community into aerobic granules. ISME J. 2014;8(6): 1186-97. https://doi.org/10.1038/ismej.2013.240.

9. Tay JH, Liu QS, Liu Y. The role of cellular polysaccharides in the formation and stability of aerobic granules. Lett Appl Microbiol. 2001;33(3):222-6. https://doi.org/10.1046/j.1472-765x.2001.00986.x.

10. Matz C, McDougald D, Moreno AM, Yung PY, Yildiz FH, Kjelleberg S. Biofilm formation and phenotypic variation enhance predation-driven persistence of Vibrio cholerae. P Natl Acad Sci USA. 2005;102(46):16819-24. https://doi. org/10.1073/pnas.0505350102.

11. Rychert K, Neu T. Protozoan impact on bacterial biofilm formation. In: Biological Letters, vol. 47; 2010. p. 3.

12. Sun S, Kjelleberg $S, M c D o u g a l d ~ D$. Relative contributions of Vibrio polysaccharide and quorum sensing to the resistance of Vibrio cholerae to predation by heterotrophic protists. PLoS One. 2013;8(2):e56338. https://doi. org/10.1371/journal.pone.0056338.

13. Brown MR, Baptista JC, Lunn M, Swan DL, Smith SJ, Davenport RJ, et al. Coupled virus - bacteria interactions and ecosystem function in an engineered microbial system. Water Research. 2019;152:264-73. https://doi. org/10.1016/j.watres.2019.01.003.

14. Khan MA, Satoh H, Katayama H, Kurisu F, Mino T. Bacteriophages isolated from activated sludge processes and their polyvalency. Water Research. 2002;36(13):3364-70. https://doi.org/10.1016/S0043-1354(02)00029-5.

15. Fan $N$, Yang $M$, Jin R, Qi R. Isolation and Genomic Characterization of an Acinetobacter johnsonii Bacteriophage AJO2 From Bulking Activated 
Sludge. Front Microbiol. 2019;10(266); doi: https://doi.org/10.3389/fmicb.201 9.00266 .

16. Liu M, Gill JJ, Young R, Summer EJ. Bacteriophages of wastewater foamingassociated filamentous Gordonia reduce host levels in raw activated sludge. Sci Rep. 2015;5(1):13754. https://doi.org/10.1038/srep13754.

17. Khairnar K, Chandekar R, Nair A, Pal P, Paunikar WN. Novel application of bacteriophage for controlling foaming in wastewater treatment plant- an eco-friendly approach. Bioengineered. 2016;7(1):46-9. https://doi.org/10.1 080/21655979.2015.1134066.

18. Barr JJ, Slater FR, Fukushima T, Bond PL. Evidence for bacteriophage activity causing community and performance changes in a phosphorus-removal activated sludge. FEMS Microbiol Ecol. 2010;74(3):631-42. https://doi.org/1 0.1111/j.1574-6941.2010.00967.x.

19. Wanner J, Ruzickova I, Krhutkova O, Pribyl M. Activated sludge population dynamics and wastewater treatment plant design and operation. Water Sci Technol. 2000;41(9):217-25. https://doi.org/10.2166/wst.2000.0210.

20. Wang Y-A, Li W, Zhuang J-L, Liu Y-D, Shapleigh JP. Bacteriophage-mediated extracellular DNA release is important for the structural stability of aerobic granular sludge. Sci Total Environ. 2020;726:138392. https://doi.org/10.1016/j. scitotenv.2020.138392.

21. Li J, Ma L, Wei S, Horn H. Aerobic granules dwelling vorticella and rotifers in an SBR fed with domestic wastewater. Separation Purification Technol. 2013; 110:127-31. https://doi.org/10.1016/..seppur.2013.03.022.

22. Lemaire R, Webb RI, Yuan Z. Micro-scale observations of the structure of aerobic microbial granules used for the treatment of nutrient-rich industrial wastewater. ISME J. 2008;2(5):528-41. https://doi.org/10.1038/ismej.2008.12.

23. Schwarzenbeck N, Erley R, Wilderer PA. Aerobic granular sludge in an SBRsystem treating wastewater rich in particulate matter. Water Sci Technol. 2004;49(11-12):41-6. https://doi.org/10.2166/wst.2004.0799.

24. Weber SD, Ludwig W, Schleifer KH, Fried J. Microbial composition and structure of aerobic granular sewage biofilms. Appl Environ Microbiol. 2007; 73(19):6233-40. https://doi.org/10.1128/AEM.01002-07.

25. Barr JJ, Cook AE, Bond PL. Granule formation mechanisms within an aerobic wastewater system for phosphorus removal. Appl Environ Microbiol. 2010; 76(22):7588-97. https://doi.org/10.1128/AEM.00864-10.

26. Liu Y, Wang Z-W. Essential Roles of Extracellular Polymeric Substances in Aerobic Granulation. In: Liu Y, editor. Wastewater Purification: Aerobic Granulation in Sequencing Batch Reactors (1st ed.). Boca Raton: CRC Press; 2008. p. 181-194.

27. Secor PR, Sweere JM, Michaels LA, Malkovskiy AV, Lazzareschi D, Katznelson E, Rajadas J, Birnbaum ME, Arrigoni A, Braun KR, Evanko SP, Stevens DA, Kaminsky W, Singh PK, Parks WC, Bollyky PL. Filamentous bacteriophage promote biofilm assembly and function. Cell Host Microbe. 2015;18(5):54959. https://doi.org/10.1016/j.chom.2015.10.013.

28. Webb JS, Lau M, Kjelleberg S. Bacteriophage and phenotypic variation in Pseudomonas aeruginosa biofilm development. J Bacteriol. 2004;186(23): 8066-73. https://doi.org/10.1128/jb.186.23.8066-8073.2004.

29. McArdle BH, Anderson MJ. Fitting multivariate models to community data: a comment on distance-based redundancy analysis. Ecology. 2001; 82(1):290-7. https://doi.org/10.1890/0012-9658(2001)082[0290:FMMTCD]2. $0 . \mathrm{CO} ; 2$.

30. Inaba T, Hori T, Sato Y, Aoyagi T, Hanajima D, Ogata A, Habe H. Eukaryotic microbiomes of membrane-attached biofilms in membrane mioreactors analyzed by high-throughput sequencing and microscopic observations. Microbes Environ. 2018;33(1):98-101. https://doi.org/10.1264/jsme2.ME17112.

31. Xie C, Goi CLW, Huson DH, Little PFR, Williams RBH. RiboTagger: fast and unbiased 16S/18S profiling using whole community shotgun metagenomic or metatranscriptome surveys. BMC Bioinformatics. 2016;17(19):508. https:// doi.org/10.1186/s12859-016-1378-x.

32. Gonzalez-Gil G, Holliger C. Dynamics of microbial community structure of and enhanced biological phosphorus removal by aerobic granules cultivated on propionate or acetate. Appl Environ Microbiol. 2011;77(22): 8041-51. https://doi.org/10.1128/AEM.05738-11.

33. Lemaire R, Yuan Z, Blackall LL, Crocetti GR. Microbial distribution of Accumulibacter spp. and Competibacter spp. in aerobic granules from a lab-scale biological nutrient removal system. Environ Microbiol. 2008;10(2): 354-63. https://doi.org/10.1111/j.1462-2920.2007.01456.x.

34. Oehmen A, Yuan Z, Blackall LL, Keller J. Comparison of acetate and propionate uptake by polyphosphate accumulating organisms and glycogen accumulating organisms. Biotechnol Bioeng. 2005;91(2):162-8. https://doi.org/10.1002/bit.20500.
35. Cydzik-Kwiatkowska A, Zielinska M. Bacterial communities in full-scale wastewater treatment systems. World J Microbiol Biotechnol. 2016;32(4):66. https://doi.org/10.1007/s11274-016-2012-9.

36. Yu K, Zhang T. Metagenomic and metatranscriptomic analysis of microbial community structure and gene expression of activated sludge. PLoS One. 2012;7(5):e38183. https://doi.org/10.1371/journal.pone.0038183.

37. Ploss M, Kuhn A. Kinetics of filamentous phage assembly. Phys Biol. 2010; 7(4):045002. https://doi.org/10.1088/1478-3975/7/4/045002.

38. Ren $T T, Y u H Q, L i X Y$. The quorum-sensing effect of aerobic granules on bacterial adhesion, biofilm formation, and sludge granulation. Appl Microbiol Biotechnol. 2010;88(3):789-97. https://doi.org/10.1007/s00253-01 0-2796-8.

39. Liu Y, Tay J-H. State of the art of biogranulation technology for wastewater treatment. Biotechnol Adv. 2004;22(7):533-63. https://doi.org/10.1016/j. biotechadv.2004.05.001.

40. Madoni P. Protozoa in activated sludge. In: Bitton G, editor. Encyclopedia of Environmental Microbiology. New York: Wiley; 2003. https://doi.org/10.1002/ 0471263397.env057.

41. Madoni P. A sludge biotic index (SBI) for the evaluation of the biological performance of activated sludge plants based on the microfauna analysis. Water Res. 1994;28(1):67-75. https://doi.org/10.1016/0043-1354(94)90120-1.

42. Rodriguez-Perez S, Fermoso FG, Arnaiz C. Influence of different anoxic time exposures on active biomass, protozoa and filamentous bacteria in activated sludge. Water Sci Technol. 2016;74(3):595-605. https://doi.org/1 0.2166/wst.2016.247.

43. Dopheide A, Lear G, Stott R, Lewis G. Preferential feeding by the ciliates Chilodonella and Tetrahymena spp. and effects of these protozoa on bacterial biofilm structure and composition. Appl Environ Microbiol. 2011; 77(13):4564-72. https://doi.org/10.1128/aem.02421-10.

44. Walczyńska A, Sobczyk M, Fiałkowska E, Pajdak-Stós A, Fyda J, Wiąckowski K. Interaction between a bacterivorous ciliate Aspidisca cicada and a rotifer Lecane inermis: Doozers and Fraggles in aquatic flocs. Microb Ecol. 2018; 75(3):569-81. https://doi.org/10.1007/s00248-017-1036-5.

45. Böhme A, Risse-Buhl U, Küsel K. Protists with different feeding modes change biofilm morphology. FEMS Microbiol Ecol. 2009;69(2):158-69. https://doi.org/10.1111/j.1574-6941.2009.00710.x.

46. Fried J, Lemmer $\mathrm{H}$. On the dynamics and function of ciliates in sequencing batch biofilm reactors. Water Sci Technol. 2003;47(5):189-96. https://doi. org/10.2166/wst.2003.0316.

47. de Kreuk MK, Kishida N, Tsuneda S, van Loosdrecht MCM. Behavior of polymeric substrates in an aerobic granular sludge system. Water Res. 2010; 44(20):5929-38. https://doi.org/10.1016/j.watres.2010.07.033.

48. Ratsak $\mathrm{CH}$, Kooi BW, van Verseveld HW. Biomass reduction and mineralization increase due to the ciliate Tetrahymena pyriformis grazing on the bacterium Pseudomonas fluorescens. Water Sci Technol. 1994;29(7):11928. https://doi.org/10.2166/wst.1994.0322.

49. Arregui L, Linares M, Pérez-Uz B, Guinea A, Serrano S. Involvement of crawling and attached ciliates in the aggregation of particles in wastewater treatment plants. Air Soil Water Res. 2008;1:ASWR.S752; doi: https://doi.org/1 0.4137/aswr.s752.

50. Yun G, Lee H, Hong Y, Kim S, Daigger GT, Yun Z. The difference of morphological characteristics and population structure in PAO and DPAOgranular sludges. J Environ Sci. 2019;76:388-402. https://doi.org/10.101 6/j.jes.2018.06.003

51. Shao Y, Chung BS, Lee SS, Park W, Lee S-S, Jeon CO. Zoogloea caeni sp. nov., a floc-forming bacterium isolated from activated sludge. Int J Syst Evol Microbiol. 2009;59(3):526-30. https://doi.org/10.1099/ijs.0.65670-0.

52. Ismail MH. The role of bacteriophages in mixed microbial communities and populations of Pseudomonas aeruginosa. In: Doctor of Philosophy. Singapore: Nanyang Technological University; 2019. p. 255.

53. Chan SH. Predator-prey interactions in aerobic granulation systems. In: Doctor of Philosophy (IGS). Singapore: Nanyang Technological University; 2018. p. 228.

54. Zhou Y, Ganda L, Lim M, Yuan Z, Kjelleberg S, Ng W. Free nitrous acid (FNA) inhibition on denitrifying poly-phosphate accumulating organisms (DPAOs). Appl Microbiol Biotechnol. 2010;88(1):359-69. https://doi.org/10.1007/s002 53-010-2780-3.

55. Smolders GJF, van der Meij J, van Loosdrecht MCM, Heijnen JJ. Model of the anaerobic metabolism of the biological phosphorus removal process: stoichiometry and pH influence. Biotechnol Bioeng. 1994;43(6):461-70. https://doi.org/10.1002/bit.260430605. 
56. Eaton AD, Franson MAH, Association APH, Association AWW, Federation WE. Standard Methods for the Examination of Water and Wastewater. Washington, DC: American Public Health Association; 2005.

57. Bushnell B. BBMap short-read aligner, and other bioinformatics tools; 2015.

58. Li D, Luo R, Liu CM, Leung CM, Ting HF, Sadakane K, et al. MEGAHIT v1.0: A fast and scalable metagenome assembler driven by advanced methodologies and community practices. Methods (San Diego, Calif). 2016; 102:3-11. https://doi.org/10.1016/j.ymeth.2016.02.020.

59. Hyatt D, Chen G-L, LoCascio PF, Land ML, Larimer FW, Hauser L. Prodigal: prokaryotic gene recognition and translation initiation site identification. BMC Bioinformatics. 2010;11:119. https://doi.org/10.1186/1471-2105-11-119.

60. Fu L, Niu B, Zhu Z, Wu S, Li W. CD-HIT: accelerated for clustering the nextgeneration sequencing data. Bioinformatics. 2012;28(23):3150-2. https://doi. org/10.1093/bioinformatics/bts565.

61. Buchfink B, Xie C, Huson DH. Fast and sensitive protein alignment using DIAMOND. Nat Methods. 2015;12(1):59-60. https://doi.org/10.1038/nmeth.3176.

62. Huson DH, Beier S, Flade I, Gorska A, El-Hadidi M, Mitra S, et al. MEGAN Community edition - interactive exploration and analysis of large-scale microbiome sequencing data. PLoS Comput Biol. 2016;12(6):e1004957. https://doi.org/10.1371/journal.pcbi.1004957.

63. Langmead B, Salzberg SL. Fast gapped-read alignment with bowtie 2. Nat Methods. 2012;9(4):357-9. https://doi.org/10.1038/nmeth.1923.

64. Li H, Handsaker B, Wysoker A, Fennell T, Ruan J, Homer N, et al. The Sequence Alignment/Map format and SAMtools. Bioinformatics (Oxford, England). 2009;25(16):2078-9. https://doi.org/10.1093/bioinformatics/btp352.

65. McMurdie PJ, Holmes S. phyloseq: an R package for reproducible interactive analysis and graphics of microbiome census data. PloS one. 2013;8(4): e61217. https://doi.org/10.1371/journal.pone.0061217.

66. Green MR, Sambrook J. Precipitation of DNA with Ethanol. Cold Spring Harbor Protocols. 2016;2016(12); doi: https://doi.org/10.1101/pdb.prot093377.

67. Shimeta J, Cook PLM. Testing assumptions of the eukaryotic inhibitor method for investigating interactions between aquatic protozoa and bacteria, applied to marine sediment. Limnol Oceanogr Methods. 2011;9(7): 288-95. https://doi.org/10.4319/lom.2011.9.288.

68. Law Y, Kirkegaard RH, Cokro AA, Liu X, Arumugam K, Xie C, StokholmBjerregaard M, Drautz-Moses DI, Nielsen PH, Wuertz S, Williams RBH. Integrative microbial community analysis reveals full-scale enhanced biological phosphorus removal under tropical conditions. Sci Rep. 2016;6(1): 25719. https://doi.org/10.1038/srep25719.

69. Feng S, Tan CH, Constancias F, Kohli GS, Cohen Y, Rice SA. Predation by Bdellovibrio bacteriovorus significantly reduces viability and alters the microbial community composition of activated sludge flocs and granules. FEMS Microbiol Ecol. 2017;93(4):fix020-fix; https://doi.org/10.1093/femsec/ fix020.

70. Zachary D. Kurtz, Christian L. Müller, Emily R. Miraldi, Dan R. Littman, Martin J. Blaser, Richard A. Bonneau, Christian von Mering. Sparse and Compositionally Robust Inference of Microbial Ecological Networks. PLoS Comput Biol. 2015;11(5):e1004226.

71. Oksanen J, Blanchet FG, Friendly M, Kindt R, Legendre P, McGlinn D, et al. vegan: Community Ecology Package. In., 2.5-6 edn; 2019.

\section{Publisher's Note}

Springer Nature remains neutral with regard to jurisdictional claims in published maps and institutional affiliations.

Ready to submit your research? Choose BMC and benefit from:
- fast, convenient online submission
- thorough peer review by experienced researchers in your field
- rapid publication on acceptance
- support for research data, including large and complex data types
- gold Open Access which fosters wider collaboration and increased citations
- maximum visibility for your research: over 100M website views per year
At BMC, research is always in progress.
Learn more biomedcentral.com/submissions

